\title{
"Just-in-Place" Information for Mobile Device Interfaces
}

\author{
Jesper Kjeldskov \\ Department of Computer Science \\ Aalborg University, Denmark \\ jesper@cs.auc.dk
}

\begin{abstract}
This paper addresses the potentials of context sensitivity for making mobile device interfaces less complex and easier to interact with. Based on a semiotic approach to information representation, it is argued that the design of mobile device interfaces can benefit from spatial and temporal indexicality, reducing information complexity and interaction space of the device while focusing on information and functionality relevant here and now. Illustrating this approach, a series of design sketches show the possible redesign of an existing web and wap-based information service.
\end{abstract}

\section{Introduction}

With the launch of wap and browsers like AvantGo for PDAs, wireless information services targeted at mobile users are now beginning to emerge. Mobile technology is, however, still very much in its infancy regarding usability, network speed, display capabilities and computing performance. While the next generation of wireless devices $(3 \mathrm{G})$ promises a platform matching the performance of desktop computers, this does not in it self provide higher usability of mobile devices and applications.

Designing usable interfaces for tomorrow's mobile devices is not trivial but involves a series of challenges on human-computer interaction. Displays on mobile devices are small, means of input are limited and use-contexts are very dynamic. The usability of mobile information services consequently suffers from interfaces being very compact and cluttered with information, thus demanding the user's full attention. In mobile use-contexts (e.g. walking down the street or driving a car) requiring a change of focus away from activities in the real world can be problematic. If mobile devices are to have higher usability while users actually being mobile, the user interface must remain simple and the required interaction minimal. This call for new user interface design.

An example of a "minimal attention interface" is the use of audio output instead of graphical displays for GPS devices presented in [1]. Another example is the use of head-up displays in automobiles or augmented reality for mobile devices in general [2][3], allowing the users to stay focused on their physical surroundings. While these approaches focus on new means of output (sound, and transparent displays), new sources of input may contribute to minimal attention interfaces by supplying means for simplifying the user interface and reducing the demand for user interaction. 


\section{2 “Just-in-Place” Information}

One of the features of the next generation of wireless devices is the ability to access information about the user's physical location and combine this information with time. Besides facilitating spatial navigation, this feature more importantly enables the design of information services pushing information based on the continuously changing context of a mobile IT-user (see e.g. [4]). Instead of viewing the dynamic use context of mobile devices as a problem, context changes can be viewed as important means of input to mobile information services. Physical space becomes part of the interface, providing the user with information and functionality adapted to a specific location in space and time: "just-in-place" information.

It is, however, too simple to state that context sensitivity will automatically result in higher usability when this information becomes available in future $3 \mathrm{G}$ mobile devices. A number of questions have to be addressed. What are the potentials of context sensitivity in relation to HCI? How does the spatial and temporal context influence on information representation and how can general insight into this relation be transformed into specific interface design for mobile devices?

Some of these issues have already been addressed in recent literature on context awareness. See e.g. [5] and [6] for usability evaluations of context sensitive information service prototypes and [7] for a discussion on conceptualization and methodology for the design of such services based on field study analysis.

\subsection{Representing "Just-in-Place" Information}

The field of semiotics concerns the meaning and use of signs and symbols. A semiotic approach to the design of "just-in-place" information can contribute to a theoretical understanding of information representation and the design of minimal attention user interfaces, taking into account their spatial and temporal context.

From a semiotic perspective, information is viewed as representations of something else (their object). Faced with an interpreter, these representations cause a reaction or interpretation.

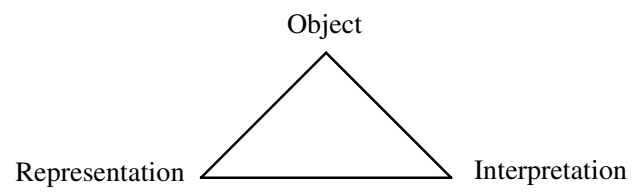

Fig. 1. Semiotics: relations between object, representation and interpretation.

The semiotics operates with three types of relationships between objects and representations: symbolic (conventional), iconic (similarity) and indexical (material/causal). Symbols and icons are ways of representing information independent of location in space and/or time like e.g. text and graphical illustrations in books or on web pages. Indexes, on the other hand, are ways of representing information with a strong relation to spatial and/or temporal location exploiting information present in the context. Indexical representations are e.g. used on signposts and information boards. 
Locating information in time and space, symbolic and iconic representations can be converted into temporal and spatial indexical representations [8]. This is illustrated in fig. 2 and 3, showing two information representations of train departures.

The timetable shown in fig. 2 exemplifies symbolic and iconic information representation with no indexicality thus being valid independent of the user's location in space and time. The electronic timetable in fig. 3 exemplifies symbolic and indexical information representation being valid (and relevant) only at a specific location at a specific time. Increasing the indexicality from the traditional to the electronic timetable results in a significant reduction of information and need for interaction. Instead of having to look up departures from a specific location at a specific time (fig. 2), the user is presented with simple information adjusted to his physical location at the present time (fig. 3). Similar examples are numerous, but prohibited due to limited space.

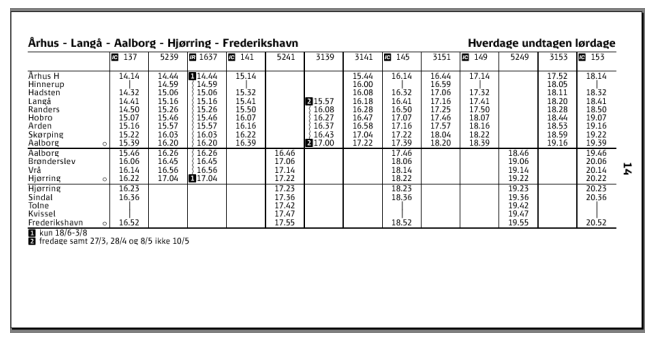

Fig. 2. Traditional timetable: symbolic representation with no indexicality.

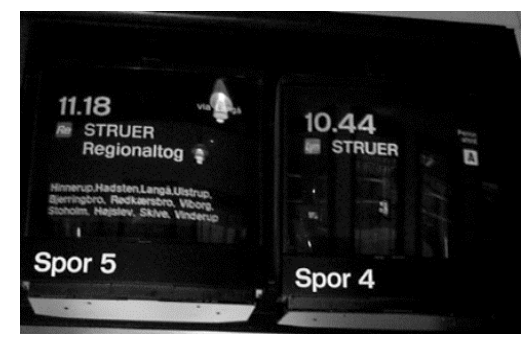

Fig. 3. Electronic timetable: spatial and temporal indexicality

\section{Designing an Indexical Interface for Mobile Devices}

Developing prototypes that actually works and can be evaluated in real mobile use contexts is of huge importance to mobile HCI research. Discussing principles of context sensitive interface design, however, does not always require actual implementation. For the purpose of illustrating the principle of indexical interfaces for mobile devices, I have redesigned an existing information service using design sketches and scenarios. As subject for redesign I choose an information service for local cinemas as this service requires the user to browse movies in relation to time and place and supports mobile access via wap.

Using the website (fig. 4a), the user specifies day of week (temporal context) and is presented with a list of all movies on this day (even if in the past) at affiliated theatres. Accessing the service from a wap phone (fig. 4b, 4c), the user specifies a cinema (spatial context) and is presented with a list of movies. Selecting a title reveals playing times. While the website is straightforward to use, the wap-site requires a lot of clicking due to the division of information into a large number of sub-pages. 


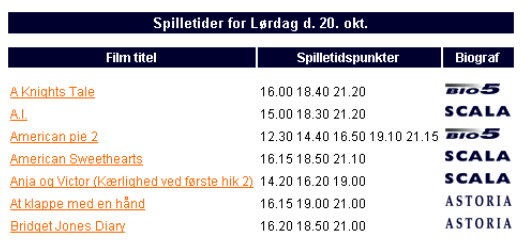

a) Web: "Movies playing tonight"

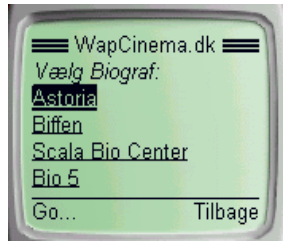

b) Wap: "Choose Cinema"

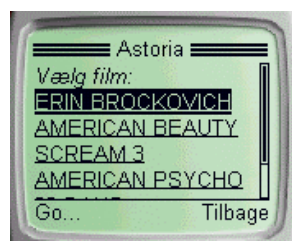

c) Wap: "Choose movie"

Fig. 4. An existing cinema information system for web (a) and wap (b and c)

\subsection{AhndexicalMobile DeviceInterface}

Figure 5 illustrates a spatial and temporal indexical representation of information designed to be available only when entering a specific cinema, providing information about movies playing here, tonight. This approach provides a limited interaction space of information meaningful only at specific spatial locations typically visited in a pre-defined sequence. Compared to the non-indexical interfaces of the web and wapsite, this design requires minimal attention and interaction. Accentuating movies playing within a limited window of time relatively increases temporal indexicality (a). Adding information about how to get to the specific halls from the user's present location increases spatial indexicality (b). The indexical interface is far less cluttered with information compared to web and wap interfaces as the user is only required be present at the cinema and select a movie from a limited list of choices.
a) Entering cinema
b) Finding Hall A
c) Outside Hall A
d) Inside Hall A
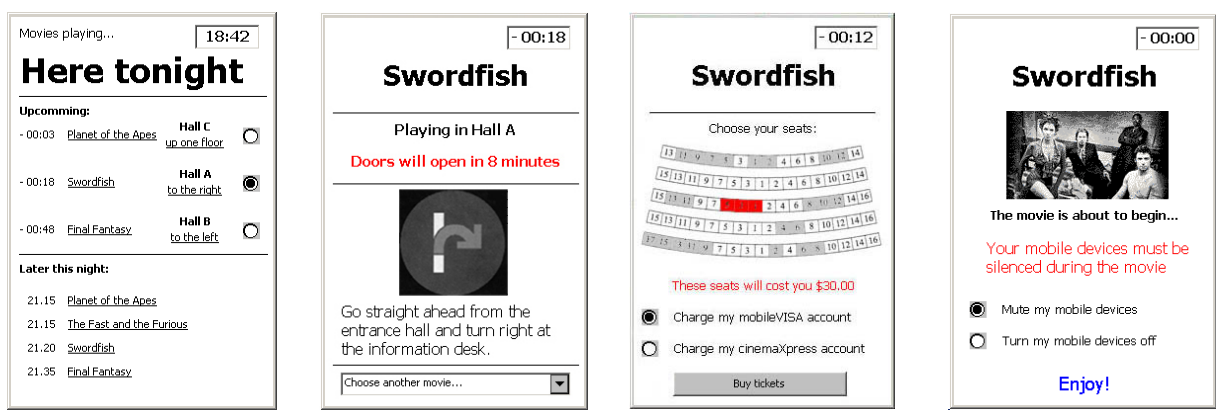

Fig. 5. A series of spatially and temporally indexical "just-in-place" interfaces for cinema information system.

One of the fundamental ideas of "just-in-place" information is that the content of the device changes when the context changes. A context sensitive mobile information service would thus typically consist of a series of indexical interfaces available at just the right places. The mobile cinema information service could involve the following use scenario and indexical interfaces: Having selected a movie (a), the device displays direction instructions (b). The representation of time changes from absolute to relative, increasing temporal indexicality. Outside Hall A, the user selects seats and buys tickets (c). When the movie begins, the user's mobile devices are silenced (d). 


\section{Conclusions and Further Work}

Interface design for mobile devices can be simplified by increasing spatial and temporal indexicality, allowing information to be left out from the interface when present in the context. Simplifying the user interface on the basis of context reduces demands for user interaction and contributes to less required attention.

For further insight into the potentials of indexical interfaces, the presented design should be implemented and made subject for comparative usability evaluations. Such evaluations should preferably take place in context. A general test bed facilitating real world usability evaluations of information services based on location aware mechanisms embodying ideas such as the presented is currently being developed.

Finally, it should be noted that context is more than just space and time. Information about the user's tasks and activity etc. may contribute to further simplification of interfaces and required interaction. For interesting research on the use of contextual sensors for mobile interaction see [9].

\section{References}

1. Holland, S. and Morse, D. R.: Audio GPS: spatial audio in a minimal attention interface. Proceedings of MobileHCI, IHM-HCI, Lille, France (2001)

2. Feiner S., MacIntyre B., Höllerer T.: Wearing It Out: First Steps Towards Mobile Augmented Reality Systems in Onto, Y. and Tamura, H. (eds.): Mixed reality - Merging Real and Virtual Worlds, Springer-Verlag, London (1999)

3. Kjeldskov J.: Lessons From Being There: Interface Design For Mobile Augmented Reality. To appear in Qvortrup L. (ed.) Virtual Applications: Applications With Virtual Inhabited 3D Worlds, Springer-Verlag, London (2003)

4. Cheverst, K. et al.: Investigating Context-aware Information Push vs. Information Pull to Tourists. Proceedings of MobileHCI, IHM-HCI, Lille, France (2001)

5. Cheverst, K. et al.: Using Context as a Crystal Ball: Rewards and Pitfalls. In Personal and Ubiquitous Computing vol. 5:8-11, Springer-Verlag, London (2001)

6. Schmidt A. et al.: Context-Aware Telephony over WAP. In Personal and Ubiquitous Computing vol. 4:225-229, Springer-Verlag, London (2000)

7. Andersen, P. B. and Nowack, P.: The Space is the Computer. To appear in Qvortrup L. (ed.): Virtual Applications: Applications With Virtual Inhabited 3D Worlds, SpringerVerlag, London (2003)

8. Andersen, P. B.: Pervasive Computing and Space. Proceedings of IFIP WG8.1. Montreal, Quebec Concordia University, Pp. 106-125 (2001)

9. Hinckley K., Pierce J., Sinclair M., Horvitz, E.: Sensing Techniques for Mobile Interaction. In Symposium on User Interface Software and Technology, CHI Letters 2(2):91-100 (2000) 